\title{
EMBRACE: First Experimental Results with the Initial $10 \%$ of a 10,000 Element Phased Array Radio Telescope *
}

\author{
S.J. Wijnholds ${ }^{1}$, G.W. Kant ${ }^{1}$, E. van der Wal ${ }^{1}$, P. Benthem ${ }^{1}$, M. Ruiter ${ }^{1}$, P. Picard ${ }^{2}$, S.A. Torchinsky ${ }^{2}$, \\ S. Montebugnoli ${ }^{3}$, and R. Keller ${ }^{4}$ \\ 1 ASTRON, Oude Hoogeveensedijk 4, NL-7991 PD Dwingeloo, The Netherlands \\ 2 Station de radioastronomie de Nançay, 18330 Nançay, France \\ 3 INAF-Instituto di Radioastronomia, Via P. Gobetti 101, 40129 Bologna, Italy \\ 4 Max-Planck-Institut für Radioastronomie, Auf dem Hügel 69, 53121 Bonn, Germany
}

\begin{abstract}
The Electronic Multi-beam Radio Astronomy Concept (EMBRACE) is the first full-scale prototype of an SKA phased array station. This single polarization demonstrator operates between 500 and $1500 \mathrm{MHz}$. The station that is currently being built at the site of the Westerbork Synthesis Radio Telescope, will ultimately consist of 144 tiles with 72 single polarization Vivaldi antennas each, giving a total of $\sim 10^{4}$ elements. The station is equipped with a LOFAR station backend with 192 input channels, which can be exploited for real-time digital beam forming in 248 frequency channels of $195 \mathrm{kHz}(\sim 48 \mathrm{MHz}$ total bandwidth) and correlation in a single $195 \mathrm{kHz}$ frequency channel. In this paper we present the results from a number of system validation measurements and the first fringes on an astronomical source, the sun. These initial measurements indicate a system temperature between $103 \mathrm{~K}$ and $117 \mathrm{~K}$. These results corroborate the feasibility and applicability of phased array technology at frequencies below $1500 \mathrm{MHz}$. They also provide valuable insights for the aperture array verification program, the next step in aperture array development towards the SKA.
\end{abstract}

\section{Introduction}

The Electronic Multi-beam Radio Astronomy Concept (EMBRACE) is the first full-scale prototype of an SKA phased array station. EMBRACE is a single polarization demonstrator operating between $500 \mathrm{MHz}$ and $1500 \mathrm{MHz}$. The antenna has been designed for two polarizations, to include all electromagnetic effects. The station that is currently being built at the site of the Westerbork Synthesis Radio Telescope (WSRT) (Kant et al. 2010), will ultimately consist of 144 tiles with 72 receive channels each, giving a total of $\approx 10^{4}$ active elements. A similar 80-tile station will be constructed at the site of the Nançay radio astronomy facility.

In this paper we present results obtained using the hardware that was available by the end of December 2009 in Westerbork as described in more detail in the next section. These include validation of the analog beamforming using Afristar, an interferometric measurement on GPS Satellite SVN49 and the first fringes observed with EMBRACE on an astronomical source, the sun. The last observation indicates that the system temperature lies between $103 \mathrm{~K}$ and $117 \mathrm{~K}$. Furthermore, the redundancy in the array may be exploited for calibration purposes. This demonstrates the feasibility and applicability of phased array technology at the EMBRACE operating frequencies and provides a nice starting point for more detailed testing within the aperture array verification program, the next phase of the aperture array development path towards the SKA.

* This work was supported by the European Commission Framework Program 6, Project SKADS, Square Kilometer Array Design Studies (SKADS), contract no. 011938.

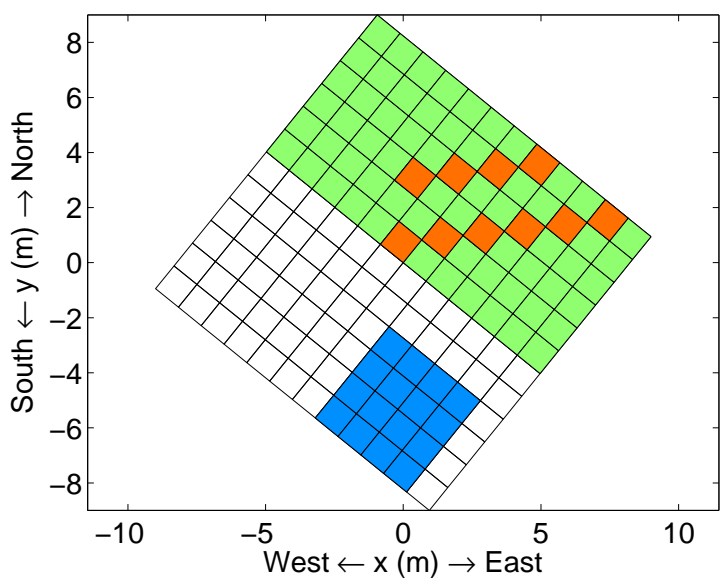

Fig. 1: EMBRACE configuration in Westerbork. The colored tiles are installed and the 10 tiles used for the fringe experiments in this paper and the $4 \times 4$ array of tiles equipped with high pass filter have been indicated.

\section{Measurement setup}

By the end of October 2009, 72 out of 144 tiles were installed at the WSRT site in a $6 \times 12$ regular array as shown in Fig. 1 . To minimize the antenna sensitivity towards the nearby radio and television tower in Smilde, the main diagonals of the tiles make an angle of about $6^{\circ}$ with the quarters of the compass. The active antennas are roughly oriented North-South. Each tile consists of 72 antennas distributed over six hexboards. The signals from these hexboards are combined in the center board, 
which provides two independent beams. A more detailed description of the frontend is provided by Ruiter \& Van der Wal (2009).

Each beam will be connected to a control and down conversion (CDC) board, which mixes the frequency range of interest to the $110-190 \mathrm{MHz}$ interval (Bianchi 2010). For the experiments presented in this paper, $16 \mathrm{CDC}$ boards were available. Initially, we selected 10 tiles on two uniform linear arrays (ULA) with roughly East-West orientation consisting of four and six tiles respectively. These tiles are indicated in Fig. 1.

During the roll-out of the 72 tiles at the WSRT site, the power spectrum at the output of the different components in the analog signal path was checked using a spectrum analyzer. From these system tests, we learned that the IP3 of the beam former chip was too high given the presence of strong DVB-T transmissions near the WSRT site. At this moment, the RFI situation at the Nançay site is much better, but digital video broadcasting is currently being rolled out throughout France and will start to affect the Nançay site within one year. This problem can be solved by including a $950 \mathrm{MHz}$ high pass filter in the signal path before the beam former chip. Sixteen still-to-be-installed tiles were modified accordingly and arranged in a $4 \times 4$ array to allow observations that are not affected by intermodulation. These tiles became available by the end of December 2009 and were used to produce the multi-tile Afristar images in this paper. Their position has been indicated in Fig. 1.

The output channel of a CDC board is connected to a digitizer board, reused from LOFAR. A full backend with 192 receive channels is installed at the WSRT site. This backend is equipped with FPGA based digital processing hardware capable of beamforming two times $48 \mathrm{MHz}$ bandwidth and correlating all 192 input channels in a single $195 \mathrm{kHz}$ wide frequency channel. It can also produce autocorrelation statistics of each signal path and of the beamformed signal with $195 \mathrm{kHz}$ frequency resolution. The integration time can be specified in integral multiples of one second. A detailed description of the digital processing is provided by Picard et al. (2010).

\section{Tile beam former validation}

The analog beamformer was validated by scanning the beam of one of the first ten tiles over the sky at $1475.25 \mathrm{MHz}$. This is within the operating range of the geostationary satellite Afristar $(1469-1481 \mathrm{MHz})$, which is located at $(a z, e l)=\left(162.1^{\circ}, 28.1^{\circ}\right)$ corresponding to direction cosines $(l, m)=(0.27,-0.84)$ as seen from the WSRT site. The resulting image, shown in Fig. 2, clearly shows the image of Afristar at the expected location as well as its grating lobe close to the northern horizon. This indicates that the analog beamformer is working correctly.

The image also shows a few artefacts. The most visible artefacts are the bright blob in the center of the image and the ripples running over the image from Northwest to Southeast. These features are produced by an interplay of several effects:

Intermodulation As discussed in the previous section, the IP3 of the beamformer chip turned out to be too high to prevent intermodulation products of the DVB-T signals from

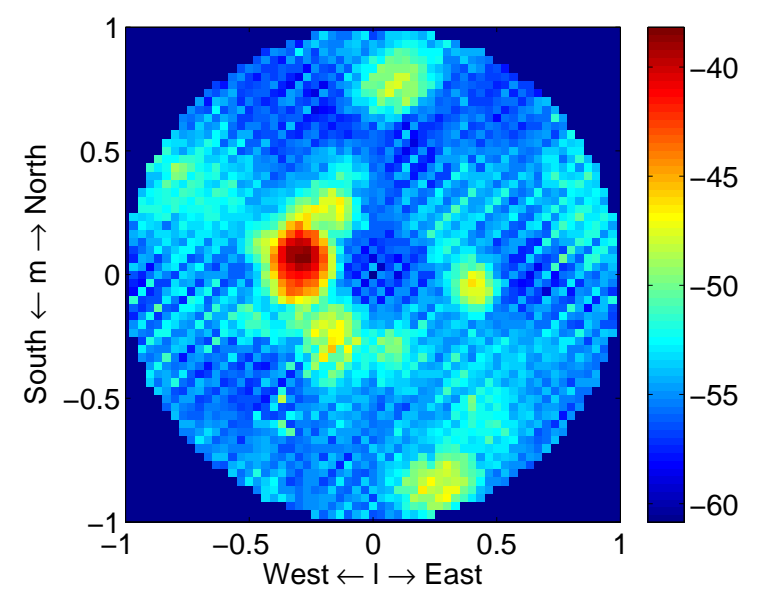

Fig. 2: Image obtained by a sky scan on an $(l, m)$-grid with 0.05 resolution at $1475.25 \mathrm{MHz}$. The integration time per pointing was $1 \mathrm{~s}$. The image shows Afristar close to the southern horizon at the correct loca- $\int$ tion, but also shows that the array is suffering from several detrimental effects. The color scale is logarithmic $(\mathrm{dB})$.

the nearby radio and television tower in Smilde from appearing over the whole operating frequency range.

LO power tuning By careful measurements at different points in the LO distribution system, we achieved a considerable improvement in gain and noise performance after this measurement.

Reflections The EMBRACE tiles are placed between telescopes 4 and 5 of the WSRT interferometer. A simple experiment with the beam pointed at telescope 4 demonstrated that the pointing of that telescope has considerable impact on the signal levels observed by the tiles.

Edge effects The image shown in Fig. 2 was made with a tile at the edge of the array. Later images produced using tiles which are fully enclosed by other tiles did not show the ripple. For phased array stations, size does matter.

We have made considerable progress on the first two issues since this initial observation. This can be demonstrated using the sixteen tiles equipped with a $950 \mathrm{MHz}$ high pass filter to mitigate the intermodulation products. These tiles were arranged in a $4 \times 4$ grid allowing us to reproduce Fig. 2 with arrays of $2 \times 2,3 \times 3$ and $4 \times 4$ tiles. The tiles were scanned over a regular $(l, m)$-grid with steps of 0.02 . For each pointing, the output signals of all tiles were correlated with $1 \mathrm{~s}$ integration and stored to disc. The advantage of correlation over beam forming is, that we can apply different beam forming schemes and produce images with and without calibration. We also increased the resolution in the image demonstrating that an all sky map may be produced by mosaicing.

The results shown in Fig. 3 were obtained by imaging on a $5 \times 5$ grid with a resolution of 0.004 around each pointing. The array was calibrated based on a single $1 \mathrm{~s}$ observation on Afristar before the sky scan was made. The data model for the covariance matrix $\mathbf{R}$ obtained in this calibration observation is

$$
\mathbf{R}=\mathbf{G a} \sigma \mathbf{a}^{H} \mathbf{G}^{H}+\sigma_{n} \mathbf{I},
$$



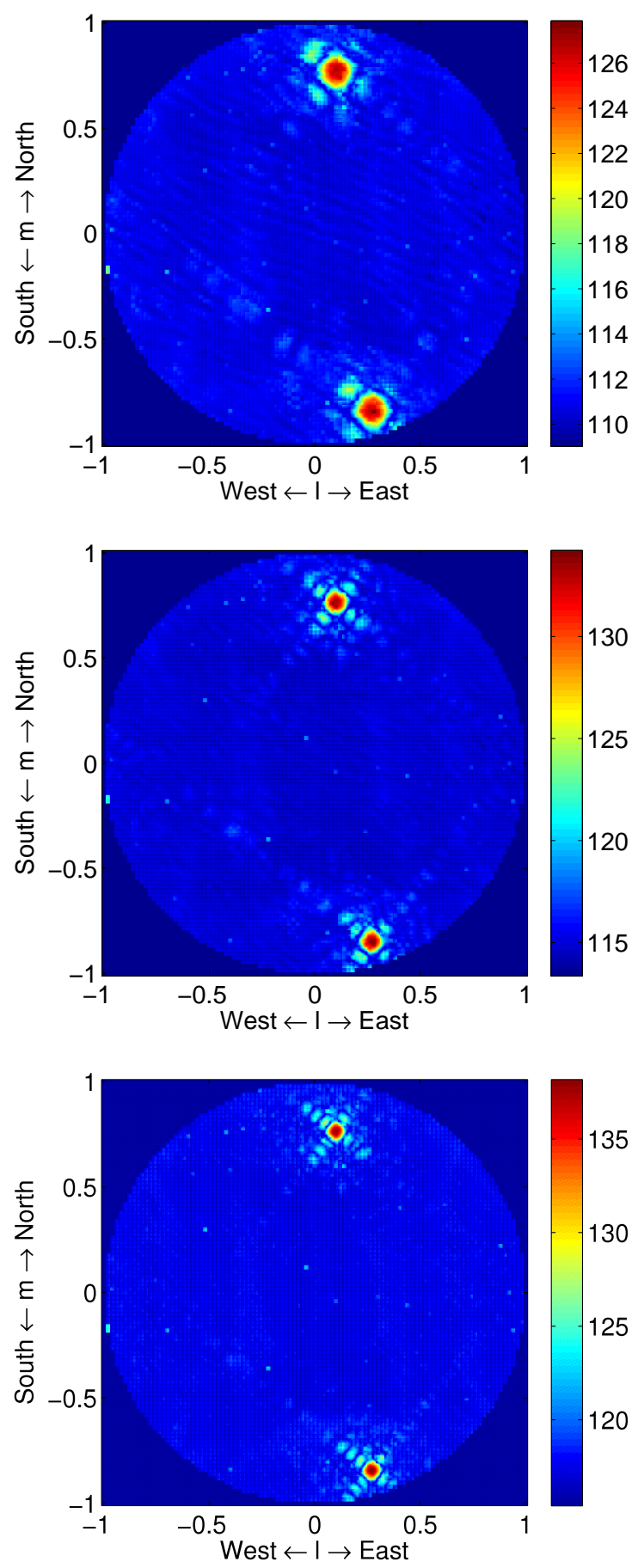

Fig. 3: Image obtained by scanning at $1475.25 \mathrm{MHz}$ with a $2 \times 2$ (top), $3 \times 3$ (middle) and $4 \times 4$ (bottom) array of tiles with 0.02 resolution using interpolation with imaging techniques to a resolution of 0.004 . The integration time per pointing was $1 \mathrm{~s}$. The images show Afristar at the correct location with increasing resolution and sensitivity. The color scale is logarithmic $(\mathrm{dB})$.

where $\mathbf{G}=\operatorname{diag}(\mathbf{g})$ is a diagonal matrix with the unknown complex valued gains of the individual signal paths on its main diagonal, $\mathbf{a}$ is the array response vector towards Afristar containing phasors describing the geometrical delays between the tiles, $\sigma$ is the source power of Afristar and $\sigma_{n}$ is the system noise power of the individual tiles, which is assumed equal for all tiles and uncorrelated between tiles. Since Afristar is the single dominant source on the sky, we can follow the same approach as used by Wijnholds et al. (2004) to calibrate LOFAR's initial test station on a RFI source, i.e. compute

$$
\mathbf{g}^{H} \mathbf{g}=\mathbf{R} \oslash\left(\mathbf{a}^{H} \mathbf{a} \sigma\right)
$$

where $\oslash$ denotes the element wise division of two matrices and obtain $\mathbf{g}$ using an Eigenvalue decomposition.

Figure 3 shows the results for the $2 \times 2$ array in the center of the $4 \times 4$ array, for the $3 \times 3$ array in the South corner and for the full $4 \times 4$ array. The images show a gradually decreasing array beam width as expected. The SNR does not improve at the $\Omega$ expected rate. A possible explanation is that, as the images also show, the sky noise is structured, implying that it cannot just be treated as spatially white Gaussian noise. Such structures have been observed with the thousand element array (ThEA), the precursor of EMBRACE (Wijnholds 2003). For ThEA, similar structures could be explained by grating responses pointing towards nearby trees with a temperature close to $300 \mathrm{~K}$ instead of to the sky with a temperature of about $3 \mathrm{~K}$. As a result, the contribution of the environment to the noise is structured and produces features in the image.

\section{Fringe measurement on the sun}

On October 28, 2009 the first ten tiles were connected to the LOFAR backend for the first interferometric solar observations with EMBRACE. Solid engineering tests have made it possible that a solar fringe was already measured in the first test observation. This is very reassuring in view of the system integration of even larger prototype systems and ultimately the SKA. At the end of the morning the tiles were pointed one hour ahead of the sun for a drift scan through the tile beams. Some of the resulting fringes are shown in Fig. 4. The observation was done in a single $195 \mathrm{kHz}$ channel at $1179 \mathrm{MHz}$ with $10 \mathrm{~s}$ integration.

The curves show the correlations of the tile at the indicated distance from the first tile of the 4-element ULA (dashed) and the 6-element ULA (solid). The width of the envelope of these fringes has a size that corresponds to the beam width of a single tile, as expected. The amplitude of this envelope allows us to determine the sensitivity, expressed as the ratio of effective area $A_{e}$ over the system temperature $T_{\text {sys }}$, towards the sun at $(a z, e l)=\left(178.0^{\circ}, 23.9^{\circ}\right)$ using

$$
\frac{A_{e}}{T_{\text {sys }}}=\frac{2 k_{b}}{S_{\text {sun }}} \frac{P_{\text {sun }}}{P_{n}+P_{\text {sun }}},
$$

where $S_{\text {sun }}$ is the flux of the sun, $P_{\text {sun }} /\left(P_{n}+P_{\text {sun }}\right)$ is the ratio of the power received from the sun over the system noise power including the self-noise from the sun and $k_{b}=$ $1.38 \cdot 10^{-23} \mathrm{WK}^{-1} \mathrm{~Hz}^{-1}$ is the Boltzmann constant. At the time of observation, the solar flux at $2.8 \mathrm{GHz}$ was about 80 . $10^{-22} \mathrm{Wm}^{-2} \mathrm{~Hz}^{-1}$, which corresponds to $14.2 \cdot 10^{-22} \mathrm{Wm}^{-2} \mathrm{~Hz}^{-1}$ at $1179 \mathrm{MHz}$ assuming that the Rayleigh-Jeans approximation holds for the solar radio spectrum at these frequencies. 


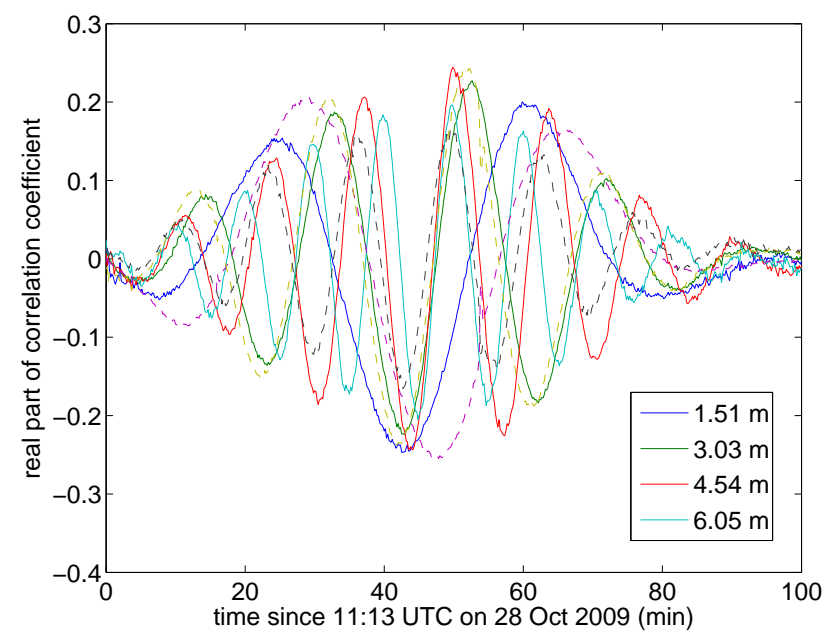

Fig. 4: Plot showing fringes on the sun at $1179 \mathrm{MHz}$ on a number of baselines with the indicated lengths from the 4-element ULA (dashed) and the 6-element ULA (solid). The observation was done in a single $195 \mathrm{kHz}$ frequency channel at $1179 \mathrm{MHz}$ with $10 \mathrm{~s}$ integration per sample.

The correlation coefficients shown in Fig. 4 are computed by dividing the power in the crosscorrelation between two tiles by the square root of the product of the autocorrelations of the corresponding tiles. From this definition, it follows that the crosscorrelation coefficient is directly related to the instantaneous SNR of the sun:

$$
\frac{P_{\text {sun }}}{\sqrt{\left(P_{n}+P_{\text {sun }}\right)^{2}}}=\frac{P_{\text {sun }}}{P_{n}+P_{\text {sun }}}=0.25 .
$$

Using Eq. (3), we thus find that the sensitivity in the direction $(a z, e l)=\left(178.0^{\circ}, 23.9^{\circ}\right)$ is $A_{e} / T_{\text {sys }}=4.86 \cdot 10^{-3} \mathrm{~m}^{2} / K$.

In an infinite dense phased array, the effective area of an element can not be larger than its physical area within the array. We can use this fact to find an upper limit for the system temperature by assuming that this also holds for the tiles that are fully enclosed by other tiles in the array. The physical size of the tile is $A_{\text {tile }}=(1.06 \mathrm{~m})^{2}=1.125 \mathrm{~m}^{2}$, which we have to multiply by the cosine of the zenith angle of the sun in the measurement $\left(66.1^{\circ}\right)$ to account for projection effects. We can thus estimate $T_{\text {sys }}$ as

$$
T_{\text {sys }}=\frac{A_{\text {tile }} \cos \left(66.1^{\circ}\right)}{A_{e} / T_{\text {sys }}}=93.8 \mathrm{~K} .
$$

This result is better than we expected. Since the tiles surrounding the tiles used for this experiment are not powered, the edge elements of the active tiles may pick up currents from neighboring tiles thus effectively enlarging their effective area. This implies that $A_{\text {tile }}$ may be somewhat larger than the physical area of a single tile. This effect could easily increase $A_{\text {tile }}$, and therefore $T_{\text {sys }}$ calculated above, by 10 to $25 \%$. This implies that the actual system temperature probably lies between $103 \mathrm{~K}$ and $117 \mathrm{~K}$. In the future, this measurement should definitely be repeated with the full array powered on to determine the impact of this edge effect more accurately. The low elevation of the sun $\left(23.9^{\circ}\right)$ might be another source of error, since the EMBRACE system was only designed for scan angles up to $45^{\circ}$ from zenith.

The LNA noise temperature is expected to be the dominant contribution to the overall system temperature. We may thus judge the reasonableness of our result by examining the expected LNA noise temperature $T_{L N A}$, which is described by (Gonzalez 1984)

$$
T_{L N A}=T_{\min }+T_{0} \frac{4 r_{N}\left|\Gamma_{a c t}-\Gamma_{o p t}\right|^{2}}{\left(1-\left|\Gamma_{a c t}\right|^{2}\right)\left|1+\Gamma_{o p t}\right|^{2}} .
$$

In this equation, $\Gamma_{o p t}$ is the reflection coefficient with optimal matching for minimal noise, $T_{\min }$ is the minimal noise temperature when the LNA is matched to $\Gamma_{o p t}, T_{0}=290 \mathrm{~K}$ by definition, $\Gamma_{a c t}$ is the active reflection coefficient, and $r_{N}$ is the normalized noise resistance. Typical values for the EMBRACE $\Omega$ LNA at $1179 \mathrm{MHz}$ are $T_{\min }=44 \mathrm{~K}, r_{N}=0.031$ and $\Gamma_{\text {opt }}=$ -0.48 . The absolute value of the active reflection coefficient of an infinite array in the direction of the sun was obtained from a full EM-simulation in HFSS and was found to be $\left|\Gamma_{a c t}\right|=0.77$,

The phase of $\Gamma_{a c t}$ is not well defined since the two reflection coefficients were obtained from two different reference planes. This implies that the value of $\left|\Gamma_{a c t}-\Gamma_{\text {opt }}\right|$ may vary from 0.29 to 1.25. In the most optimistic scenario, in which the phases of $\Gamma_{a c t}$ and $\Gamma_{o p t}$ are the same, the second term in Eq. (6) may be $\Omega$ as low as $27.5 \mathrm{~K}$. This gives a total LNA noise temperature of $71.5 \mathrm{~K}$. Adding a second stage contribution in the system of approximately $25 \mathrm{~K}$ and the sky noise contribution of $3 \mathrm{~K}$, the expected minimum system noise temperature is around $100 \mathrm{~K}$ for this specific scan angle.

Given that the accuracy of the measurement is hampered by the fact that the position of the sun was outside the scan range specification for EMBRACE and that the simulation results are difficult to interpret due to the mismatch in reference planes for the reflection coefficients, it is very reassuring that the numbers obtained from simulations and experiments are as close as they are. However, it would be nice to further improve the accuracy and confirm these numbers on different sources. Several attempts were therefore made to observe Cas A having a flux of about $2 \mathrm{kJy}$ at $1179 \mathrm{MHz}$. This should, extrapolating from the solar results, give a correlation coefficient of about $1 \%$ at the moment of transit, which happens at only $7^{\circ}$ from the zenith. Unfortunately, artifacts produced by intermodulation already appeared at $2 \%$ to $3 \%$ level. The intermodulation products were therefore overpowering Cas A, even in bands that look free of intermodulation products at first sight.

There are many redundant baselines in a dense regular phased array like EMBRACE. This can be exploited for calibration purposes as discussed by Noordam \& De Bruyn (1982), Wieringa (1992) and Noorishad et al. (2010). We therefore checked the visibilities on several redundant baselines to see whether they showed similar behavior over time. As an example, we show the phase measured on a number of redundant $1.51 \mathrm{~m}$ and $3.20 \mathrm{~m}$ baselines as function of time during the solar observation in Fig. 5. Since the array is uncalibrated, the measured phases are not the same, but it is clear from this plot that they only differ by a phase offset that could be calibrated out. The behavior of visibilities on redundant baselines 

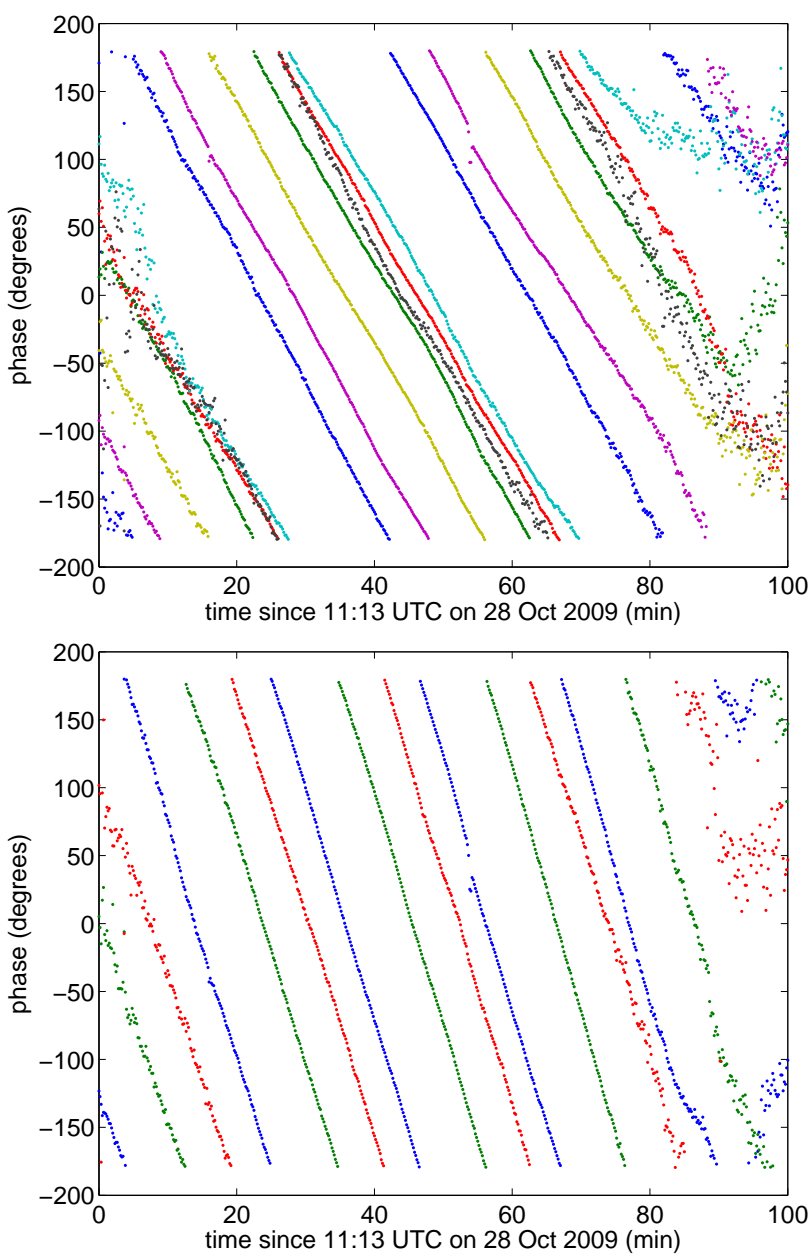

Fig. 5: Example of phase measurements during the fringe measurement on the sun obtained for a number of redundant $1.51 \mathrm{~m}$ (top) and $3.20 \mathrm{~m}$ (bottom) baselines.

looks promising, which implies that detrimental effects like mutual coupling and edge effects have a limited impact on the redundancy of the visibilities, which clears the path for exploiting it in station calibration and imaging routines.

Figure 5 shows a number of samples at the edges of the time interval for which the redundant behavior seems to break down. These are the moments at which the sun moves into and leaves the main beam respectively. When the sun is outside the main beam, the SNR of the celestial signals is so low that the intermodulation products dominate the measurements. These signals may be subject to near field multipath effects due to reflections on the nearby WSRT dishes, different IP3s of different beamformer chips and edge effects due to the low elevation of the source of the DVB-T signals causing the redundancy to break down.

\section{Interferometric detection of GPS satellites}

During one of the drift scan observations at $1179 \mathrm{MHz}$ we observed the fringes shown in Fig. 6 on a number of baselines with the indicated lengths within the 6-element ULA depicted

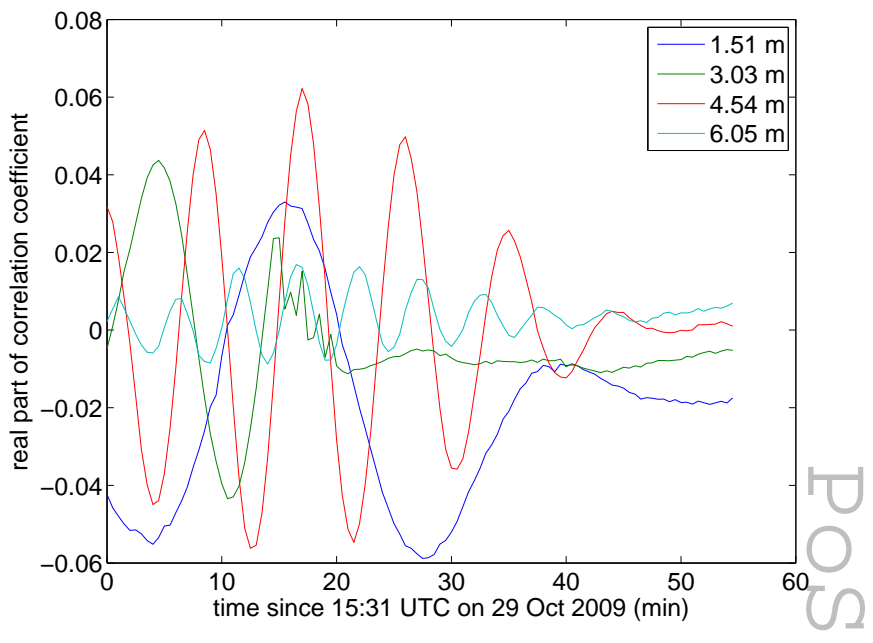

Fig. 6: Fringes measured in the GPS L5 band at $1179 \mathrm{MHz}$ from satellite SVN49. This measurement was done in a single $195 \mathrm{kHz}$ channel with $30 \mathrm{~s}$ integration time.

in Fig. 1. The fringe rate of about $22.5^{\circ} / \mathrm{h}$ is too fast for an astronomical source, which hints at a satellite signal. It turns out that on 24 March 2009 Lockheed Martin has launched GPS satellite SVN49, which includes a prototype transmitter for the GPS L5 band $(1176 \pm 12 \mathrm{MHz})$. This observation thus turned out to be the first interferometric detection of a GPS satellite with the EMBRACE system.

At the other, widely used, GPS frequencies more satellites can be detected which traverse different paths over the sky. This can be exploited for beam measurements by pointing the beam of a tile at a given position and waiting for several GPS satellites to drift through the beam following distinct paths. Such an experiment was conducted with a tile located at the Nançay site using a spectrum analyzer as reported by Olofsson et al (2010)

\section{Conclusions}

In this paper we have presented the first experimental results obtained with the EMBRACE station at the WSRT site. Although the system validation measurements indicate that we still have to tackle a few challenges imposed by the environment (presence of strong DVB-T signals, nearby WSRT dishes) before we can observe weak astronomical sources like Cas A and HI, the first fringes of the sun observed at an elevation of only $24^{\circ}$ look very promising. They indicate that the system temperature probably lies between $103 \mathrm{~K}$ and $117 \mathrm{~K}$. Moreover, the visibilities on redundant baselines showed very similar behavior in this solar observation, which is very reassuring from a station calibration perspective. Our results corroborate the feasibility and applicability of phased array technology at frequencies below $1500 \mathrm{MHz}$.

In view of further aperture array developments towards SKA, we can learn a few important lessons from these simple experiments. If the goal is to demonstrate that aperture arrays form an astronomically viable and cost and power effective solution at these frequencies, the next prototype needs to 
be build at a location that has a RFI environment similar to that of the candidate SKA sites. This will ensure that the high dynamic range design of the analog system can be tailored to the radio astronomical application instead of to the RFI environment, which will save a lot of man power and reduce the power consumption of the analog system considerably. Operation of future prototypes on such a site also helps to focus on issues that are important to astronomers like image fidelity, stability and polarization instead of putting the focus on technical issues like RFI mitigation and multipath effects that will play a role at much lower signal levels in the final SKA stations than at the EMBRACE sites.

\section{References}

Bianchi, G., EMBRACE Local Oscillator Distribution, 2010, in Proc. Wide Field Science and Technology for the SKA, Limelette, Belgium, S.A. Torchinsky et al. (eds), ASTRON, ISBN 978-90-805434-5-4

Gonzales, G., Microwave Transistor Amplifiers: Analysis and Design, Prentice-Hall Inc., Englewood Cliffs (New Jersey), USA, 1984

Kant, G.W., Wal, E. van der, Ruiter, M. and Benthem, P., The building of EMBRACE, 2010, in Proc. Wide Field Science and Technology for the SKA, Limelette, Belgium, S.A. Torchinsky et al. (eds), ASTRON, ISBN 978-90805434-5-4

Noordam, J.E. and Bruyn, A.G. de, High dynamic range mapping of strong radio sources, with application to 3 C84. Nature, vol. 299, no. 5884, pp597-600, 14 October 1982

Noorishad, P., Wijnholds, S.J., Ardenne, A. van and Hulst, J.M. van der, Applicability of Redundant Baseline Calibration on Dense Phased Arrays, 2010, in Proc. Wide Field Science and Technology for the SKA, Limelette, Belgium, S.A. Torchinsky et al. (eds), ASTRON, ISBN 978-90805434-5-4

Olofsson, H. et al., EMBRACE beam profile measurements using GPS satellites, 2010, in Proc. Wide Field Science and Technology for the SKA, Limelette, Belgium, S.A. Torchinsky et al. (eds), ASTRON, ISBN 978-90805434-5-4

Picard, P., EMBRACE station processing, 2010, in Proc. Wide Field Science and Technology for the SKA, Limelette, Belgium, S.A. Torchinsky et al. (eds), ASTRON, ISBN 97890-805434-5-4

Ruiter, M., Wal, E. van der, EMBRACE, a 10000 Element Next Generation Aperture Array Telescope, European Microwave Conference 2009, pp326-329, 29 September 2009 - 1 October 2009

Wieringa, M.H., An investigation of the telescope based calibration methods 'redundancy' and 'self-cal'. Experimental Astronomy, vol. 2, no. 4, pp203-225, 1992

Wijnholds, S.J., Evaluation and Demonstration of THEP as a Radio Astronomical Observing Facility. Master's thesis, University of Groningen, Groningen, The Netherlands, May 2003

Wijnholds, S.J., Bregman, J.D. and Boonstra, A.J., Sky Noise Limited Snapshot Imaging in the Presence of RFI with
LOFAR's Initial Test Station. Experimental Astronomy, vol. 17, nos. 1-3, pp35-42. 2004 